\title{
LINEAR THEORY OF FAST RECONNECTION AT AN X-TYPE NEUTRAL POINT
}

\author{
I. J. D. CRAIG \\ Department of Mathematics, University of Waikato, Hamilton, New Zealand \\ AND \\ A. N. MCClymont \\ Institute for Astronomy, University of Hawaii, Honolulu, HI 96822 \\ Received 1992 March 10; accepted 1992 September 3
}

\begin{abstract}
A linear theory is developed for the dissipation of the free magnetic energy in small disturbances imposed on the potential field of an X-type neutral point. An eigenmode analysis, using cylindrical coordinates centered on the neutral point, extends the work of Craig \& McClymont (1991) to include non-azimuthally symmetric perturbations on the flux function. We demonstrate that all physically significant disturbances, both reconnective and nonreconnective, decay resistively on a "fast" time scale $\sim|\ln \eta|$, where $\eta$ is the nondimensional resistivity. Thus resistive diffusion is remarkably effective as a dissipation mechanism for all classes of perturbation not only those modes which induce changes in the field topology.

The fundamental reason for the fast dissipation is the focusing of wave energy onto the neutral point by the gradient in Alfvén speed. This occurs for all types of disturbances launched from the outer boundary, but reconnective modes propagate purely radially while nonreconnective modes correspond to waves with an azimuthal motion and a smaller wave speed in the radial direction. Dissipation of reconnective disturbances takes place in a diffusion region of diameter $\approx \eta^{1 / 2}$ around the neutral point, on a time scale $\propto|\ln \eta|^{2}$, but nonreconnective perturbations decay before penetrating to the neutral point, because of the increased path lengths due to their azimuthal motion. These modes release their energy in an annulus of radius $\approx\left[\left(m^{3} /\right.\right.$ $4 k) \eta]^{1 / 2}$, where $m$ and $k$ are azimuthal and radial wavenumbers: because of their longer transit time, nonreconnective waves decay on a $|\ln \eta|^{3}$ time scale.

We conclude by discussing the significance of the linear theory within the general context of steady state and dynamic reconnection studies. It is pointed out that, dynamically, disturbances can be expected to focus explosively in the vicinity of the neutral point. This suggests the formation of a "flux pile-up" current layer in which the bulk of the magnetic energy is released as heat rather than kinetic energy of mass motion.
\end{abstract}

Subject heading: MHD

\section{INTRODUCTION}

Magnetic reconnection is the process which governs the topological simplification of complex cosmic magnetic fields. It is thought crucial in many circumstances, for example, to account for the explosive energy release in solar flares, the quiescent heating of solar and stellar coronae, and the evolution of magnetic structures in Earth's magnetosphere. A central issue is the speed of magnetic reconnection. In solar flare theory, for instance, the reconnection rate has to be "fast" to account for the violent annihilation of coronal magnetic fields on the observed flare time scale of $O(100) \mathrm{s}$, which implies that the reconnection rate can depend only weakly on the very small amount of resistivity available. In general we take "fast reconnection" to mean that the global energy dissipation rate depends only logarithmically on the plasma resistivity.

Historically, much theoretical effort has been expended in constructing approximate steady state analytic models of the reconnection process. The pioneering works of Dungey (1953), Sweet (1958), and Parker (1963), who found magnetic reconnection to be much too slow to account for solar flare energy release, led to Petschek's (1964) demonstration that neutral point reconnection could be fast, given a suitable "driven" flow topology. However, more recently, a gap has developed between analytic studies that follow the time-honored approximate steady state approach, and a new generation of theoretical simulation experiments that mimic the dynamics of the magnetic merging process. For instance, Biskamp (1986), assuming the same incompressibility condition as Petschek, presents convincing evidence - in line with previous simulation studies - that the reconnection rate is slow in contradiction to Petschek's model. Although Forbes \& Priest (1987) counter that Biskamp's boundary conditions are not compatible with those required for fast reconnection, there remains little evidence from simulations for fast Petschek-type reconnection in the literature. It is also becoming clear that not only are numerical simulations difficult to interpret within the approximate analytic framework developed to describe steady state driven reconnection (e.g., Forbes \& Priest 1987), but also that the steady state analyses have obvious limitations when it comes to understanding an intrinsically dynamic process.

It is in fact possible to obtain an analytic description of dynamic reconnection in the limit of small disturbances on an equilibrium potential field, provided that the plasma pressure is small enough (Craig \& McClymont 1991, Hassam 1991, Craig \& Watson 1992). These authors show how a description of the time-dependent relaxation of a perturbed X-type neutral point in a two-dimensional magnetic field can be developed in terms of cylindrical modes. In this formulation, topological reconnection occurs only for purely radial $(m=0)$ disturbances on the flux function. The reconnection is found to be oscillatory, with inertial overshoot of the plasma carrying more magnetic flux through the neutral point than is required 
to reach a static equilibrium. In the eigenmode description developed by Craig \& McClymont (1991), the oscillation frequency $\omega$ and decay rate $\alpha$ of the slowest decaying eigenmode are given by

$$
\omega=\frac{\pi}{|\ln \eta|}, \quad \alpha=\frac{1}{2} \omega^{2},
$$

where $\eta$ is the resistivity, and all variables are given in dimensionless units derived from the radius of the cylindrical boundary and the Alfvén speed at the boundary. The fundamental frequency is determined essentially by the travel time, $\left|\ln r_{c}\right|$, of a wave of local speed $u_{\mathrm{A}} \propto|B| \propto r$ between the outer boundary and the onset of the diffusion layer $r_{c} \approx \eta^{1 / 2}$ (Craig \& Watson 1992). The wave takes one Alfvén time to diffuse to the neutral point, so the time for a complete round trip is $\tau=$ $4\left(\frac{1}{2}|\ln \eta|+1\right)$, which for $\eta \ll 1$ gives the result (1.1) for $\omega$.

The derivation of the decay rate is more delicate, but analytic considerations confirm the $|\ln \eta|$ dependence under quite general circumstances (see the derivation of $\S 3$ ). Craig \& Watson (1992) in fact emphasize that the bulk of the energy associated with a radial disturbance generated on the Alfvén time scale at the boundary dissipates signficantly faster than the rate for the fundamental eigenmode would indicateactually within the first few bounces-as a result of rapidly decaying higher harmonics (see $\S 5.2$ ). Thus the fundamental decay rate is best interpreted as a lower limit.

The main purpose of the present paper is to extend the dynamic reconnection analysis by studying the behavior of nonradial $(m \neq 0)$ perturbations on the flux function. Unlike the purely radial $m=0$ modes, which involve topological changes in the field line connectivity, higher $m$ disturbances can decay by mechanisms other than resistive damping, for example, by viscously damped fluid motions. However, if we can show that these modes decay rapidly by resistive damping alone, then we reach the interesting conclusion that ohmic dissipation is capable of removing all the free energy, kinetic as well as magnetic, in any disturbance, with surprising efficiency.

The general problem is formulated in $\S 2$. In $\S 3$ we derive the fundamental dependence of the decay rate on the plasma resistivity using a matched asymptotic analysis valid in the limit of small $\eta$. These results are augmented by numerical computations of nonradial eigenmodes. In $\S 4$ the effects of relevant physics which cannot be treated analytically are discussed briefly, and in $\S 5$ we relate the present analysis to previous work. Finally $\S 6$ summarizes our conclusions.

\section{FORMULATION OF THE PROBLEM}

\subsection{Background and Assumptions}

We consider the relaxation of a peturbation superposed on a two-dimensional potential magnetic field containing an X-type neutral point. This potential field, being a minimum energy configuration, is the endpoint of evolution in the presence of resistive dissipation. To make the problem tractable, we consider small perturbations and linearize about the final equilibrium. The magnetic field, which we assume to be strictly two-dimensional (no field normal to the plane), is immersed in a uniform density background plasma and anchored at a perfectly conducting circular boundary at a distance $R$ from the neutral point. We identify $R$ with the length scale of a typical coronal magnetic structure. In our analysis all lengths are expressed in units of $R$, flow speeds are measured in units of the Alfvén speed, $v_{\mathrm{A}}$, at the outer boundary, and time is measured in units of $R / v_{\mathrm{A}}$. Since the magnetic pressure in the corona greatly dominates the gas pressure, we assume that the background plasma is arbitrarily compressible. We also neglect viscosity and assume uniform resistivity. Thus the problem described analytically reduces to the evolution of a highly (but not perfectly) conducting magnetized inviscid fluid in the presence of a magnetic neutral point, subject to $\boldsymbol{J} \times \boldsymbol{B}$ forces acting on its inertia.

\subsection{The Equilibrium Field}

The two-dimensional equilibrium field is described in the dimensionless formulation of Craig \& McClymont (1991) and Craig \& Watson (1992) by the flux function $\psi_{E}$, which satisfies the current-free condition

$$
\nabla^{2} \psi_{E}=0
$$

In cylindrical coordinates, solutions of equation (2.1) may be obtained by superposing the elementary solutions $r^{m} e^{ \pm i m \phi}$ where $m$ is a positive integer. The classic X-type neutral point given by

$$
\psi_{E}=-\frac{1}{2} r^{2} \cos 2 \phi
$$

corresponds to the magnetic field

$$
\boldsymbol{B}_{E}=r(\sin 2 \phi \hat{\boldsymbol{r}}+\cos 2 \phi \hat{\boldsymbol{\phi}}) .
$$

This is the simplest solution of physical interest, having the topology of two like subphotospheric dipoles as required by the Sweet-Parker flare mechanism. The higher order solutions of equation (2.1) are of greater topological complexity-the field of each individual mode is divided by separatrices into $2 m$ lobes. Since the lowest $m r$-dependence dominates near a neutral point, only perfect symmetry in the boundary conditions can allow a higher order neutral point topology than the basic X-type, in which the separatrices cross at $90^{\circ}$. In general, superpositions of the various modes result in a field containing both O-type and X-type neutral points (e.g., Watson 1991.)

\subsection{Evolution Equations}

We suppose that the equilibrium field is constantly buffeted by low-amplitude disturbances, caused, for instance, by photospheric motions or MHD instabilities in neighboring coronal magnetic structures. The key question is how fast the excess energy in the stressed field can be dissipated by resistive diffusion: this tells us the natural relaxation rate in the absence of other dissipation mechanisms.

For disturbances of small amplitude, the evolution of the magnetic field is determined by the linearized induction equation

$$
\psi=-v \cdot \nabla \psi_{E}+\eta \nabla^{2} \psi
$$

which describes advection and diffusion of the magnetic flux. Here $\psi$ is a first-order variation on the equilibrium flux function $\psi_{E}$. Disturbances which upset the intrinsic neutral point topology - that is, shift the position of the separatrix field lines on the boundary - can be dissipated only by resistive reconnection. In an ideal plasma such "reconnective perturbations" eventually "blow up" forming a singular current layer at the neutral point: for these disturbances, $\eta$, no matter how small, can never be neglected since only resistivity can avert the collapse to a singularity.

The linearized momentum equation,

$$
\dot{v}=-\nabla^{2} \psi \nabla \psi_{E}
$$


describes the motion of the plasma in response to the Lorentz force $J \hat{z} \times B_{E}$, where $J=-\nabla^{2} \psi$ is the dimensionless current density. The plasma density, which remains uniform to first order under the linearized equations, has been taken as unity. An important feature of this equation is that the acceleration is always perpendicular to the (equilibrium) field lines. It is this property that permits us to develop a modal description in which each azimuthal component is treated separately.

\subsection{Modal Representation}

Equations (2.4) and (2.5) van be combined into a single thirdorder equation for the perturbed flux function $\psi$. This equation is azimuthally invariant so we can take

$$
\psi(r, \phi, t)=\psi_{m}(r, t) e^{i m \phi},
$$

with each azimuthal component satisfying

$$
\ddot{\psi}_{m}=r^{2} \nabla^{2} \psi_{m}+\eta \nabla^{2} \psi_{m}
$$

where $\left|B_{E}\right|=r$ has been used and $\nabla^{2} \equiv(1 / r) \partial(r \partial / \partial r) / \partial r-$ $m^{2} / r^{2}$.

The boundary condition at $r=0$ follows from the boundedness of $\psi$ and the current density at the origin,

$$
\left|J_{m}\right|=\left|\nabla^{2} \psi_{m}\right| \equiv\left|\frac{\partial^{2} \psi_{m}}{\partial r^{2}}+\frac{1}{r} \frac{\partial \psi_{m}}{\partial r}-\frac{m^{2}}{r^{2}} \psi_{m}\right| .
$$

Thus we require

$$
\partial \psi_{0}(0, t) / \partial r=0(m=0), \quad \text { or } \quad \psi_{m}(0, t)=0(m \neq 0) \text {. }
$$

On the outer boundary, $r=1$, the field is line-tied, so that perturbations on the radial component of the field, $B_{r}=$ $\partial \psi / \partial \phi$, vanish for all time. Thus $\psi$ is constant along the boundary, and we can conveniently take $\psi(r=1) \equiv 0$. Since the fluid is effectively tied to the field lines in the outer region, the velocity $\boldsymbol{v}$ vanishes on $r=1$.

If line-tying is to hold throughout the evolution of a "reconnective" disturbance, the initial field must have the same distribution of normal flux on the boundary as the final potential field. Such a perturbation can be constructed by allowing "flux emergence" through the boundary in two opposite lobes of the $\mathrm{X}$, and the same amount of flux submergence in the other two lobes, then shifting the footpoints to restore the distribution of normal field. This "closes up" the angle of the $X$ (connectivity within the volume being retained), and relaxation to the potential field requires topological change. The nonradial modes, on the other hand, correspond to purely internal distortions of the field lines; the separatrices remain connected to points on the boundary $90^{\circ}$ apart. These modes therefore cannot be generated (statically) by field line manipulation on the boundary. They can obviously be generated in a dynamic manner, however, by cyclic disturbances which return the magnetic footpoints to their original positions, but leave waves propagating into the interior of the volume.

\subsection{Properties of the Modal Equation}

It is instructive to explore the physical content of the modal equation (2.7) by examining individual terms in isolation. There are three possibilities depending on the relative importance of the diffusion and advection terms. In the limit of static diffusion, $\psi_{m}=\eta \nabla^{2} \psi_{m}$, which implies Bessel function solutions

$$
\psi_{m}=J_{m}(\sqrt{\alpha / \eta} r) e^{-\alpha t}
$$

(this equation is the only instance in which $J$ does not denote current density) with $\alpha$ determining the rate of resistive decay. The decay rate associated with static diffusion is, of course, very slow for global length scales. From the condition that $\psi_{m}$ vanishes at the outer boundary $(r=1)$ we find from the asymptotic $(\alpha / \eta \gg 1)$ behavior of the Bessel function that

$$
\alpha \approx\left\{\begin{array}{cc}
m^{2} \eta & \left(m^{2} \gg n^{2} \gg 1\right) \\
\pi^{2} n^{2} \eta & \left(n^{2} \gg m^{2} \gg 1\right)
\end{array},\right.
$$

where $n$ is the number of radial nodes. Thus only very short length scales $-n$ or $m$ comparable to $1 / \sqrt{ } \eta^{1 / 2} \approx 10^{7}$ in the solar corona - are consistent with rapid static decay.

More generally, we expect equation (2.10) to apply only in the vicinity of the neutral point. Away from the origin $\left(r \gg \eta^{1 / 2}\right)$, the problem becomes advectively dominated. Dropping the dissipative term in equation (2.7) gives the wave equation $\ddot{\psi}_{m}=r^{2} \nabla^{2} \psi_{m}$ with wave speed $r$. It is readily verified that a traveling wave spiraling toward (or away from) the neutral point,

$$
\psi=e^{i( \pm \omega t \pm k s \pm m \phi)}, \quad \omega^{2}=k^{2}+m^{2}, \quad s=-\ln r
$$

provides an exact solution. This solution in which $B_{\phi}=$ $-\partial \psi / \partial r \propto 1 / r$, highlights the localization of global reconnective disturbances and the massive current build-up, $J \propto 1 / r^{2}$, as the wave approaches the origin (Craig \& Watson 1992). Buildup of the current density to an intensity of $O\left(\eta^{-1}\right)$ provides a signature for the onset of fast diffusion in a resistive plasma.

There remains one final possibility. Equation (2.7) shows that diffusion and advection can balance under the approximation $\ddot{\psi}_{m}=0$. The solution for the current density is then

$$
J(r, \phi, t) \approx g(r) e^{-r^{2} t / \eta} e^{i m \phi},
$$

where $g(r)$ is an arbitrary function which vanishes at the neutral point. This approximation - the "similarity mode" described by Hassam (1991) - has some pathological properties. We note for instance that the long time behavior suggests a persistent current density that localizes around the neutral point but does not decay. In fact this near singular structure must always be restricted to the diffusion layer, lest the approximation $\ddot{\psi}_{m}=0$ be violated. It follows that the similarity mode can never be significant energetically for a global disturbance initiated in the outer advection region.

\section{THE NONRADIAL MODES}

\subsection{Introduction}

Unlike reconnective disturbances, nonradial modes do not involve topological simplification of the magnetic field structure. Field lines can be bent, but their connections must be left intact. It follows that nonradial modes can decay by means other than resistive diffusion, for instance by viscous dissipation. However, the "focusing" effect, by which the gradient in Alfvén speed guides wavefronts unerringly toward the neutral point, may make resistive decay important for these modes, too. The oscillatory nature of the reconnection found for the $m=0$ mode suggests that the higher $m$ modes may also undergo some form of oscillatory dissipation. If resistivity turns out to be important in the damping of nonradial disturbances, these may contribute to intense heating of the plasma around the neutral point. Therefore, it is of interest to determine whether "fast" oscillatory dissipation applies to the higher $m$ modes, too.

In what follows we show that the oscillation and decay rates of the nonradial modes can be deduced from an extension of 
the boundary layer analyses given by Craig \& McClymont (1991) and Hassam (1991) for the case of reconnective $(m=0)$ disturbances. The analysis is developed in a way which allows all azimuthal modes to be treated on the same footing. Special features of the nonradial modes $(m \neq 0)$ are summarized in $\S 3.4$ and beyond.

\subsection{The Eigenmode Description}

Although a description in terms of traveling waves evolving from specified initial conditions (e.g., Craig \& Watson 1992) can yield much physical insight - and we will refer to it repeatedly for that reason-eigenmodes, precisely because of their lack of the causal element which makes the evolution picture so valuable, have the advantage of rendering results independent of initial conditions. Thus, in attempting to establish whether resistive dissipation occurs on a "fast " time scale for all possible disturbances, an eigenmode description is particularly valuable. The question of completeness of the eigenmodes is addressed in $\S 5.3$.

The development of discrete eigenmodes requires communication between all points in the spatial domain. Consider the motion of an undamped wave (2.12) in s-space, where $s=-\ln r$. The wavespeed in $s$-space is unity so, in the absence of resistivity, a traveling wave launched from the outer boundary, $s=0$, can never reach the neutral point, $s=\infty$. It follows that the system possesses a continuum of eigenvalues $k$, and that the evolution of any initial disturbance can be described by a Fourier integral over $k$ (Craig \& Watson 1992).

This picture is radically changed by the introduction of resistivity. Information from the outer field propagates to the resistive layer surrounding the neutral point, then diffuses rapidly through the origin. The communication established between the origin and the outer boundary makes possible the development of radial modal structure comprising standing wave eigenmodes. The radial wavenumbers $k$ must now be discrete to reflect the finite advection domain.

Although the heart of our analysis is couched in terms of standing waves eigenfunctions, we will also find it instructive to consider the propagation of a traveling wave as described by equation (2.12). It is clear that in $s$-space the wave travels at an angle $\tan ^{-1}(\mathrm{k} / \mathrm{m})$ to the azimuthal direction, along the path $s=(k / m) \phi$. In real space the wave packet follows the spiral trajectory

$$
r=e^{-(k / m) \phi}
$$

so that a wave packet with $k \ll m$ circulates almost azimuthally, while a wave packet with $k \gg m$ travels radially. The path length of the wave trajectory between the origin and the outer boundary then increases as the ratio $m / k$.

\subsection{Analysis of the Eigensystem}

To determine eigensolutions we set

$$
\psi_{m}(r, t)=e^{\lambda t} f(r),
$$

where $f=f_{\mathbf{R}}+i f_{\mathrm{I}}$ is a complex eigenfunction. The eigenvalue $\lambda=i \omega-\alpha$ determines the oscillation and decay rate of the mode. Writing $f^{\prime}$ for $d f / d r$, the eigensystem is given by

$$
r\left(r f^{\prime}\right)^{\prime}=\left(-\frac{\lambda^{2}}{1+\lambda \eta / r^{2}}+m^{2}\right) f, \quad f(0)=\delta_{m 0}, \quad f(1)=0 .
$$

For a sufficiently thin diffusion layer we anticipate an oscillatory standing wave of negligible decay rate. Accordingly we treat the diffusion layer as a narrow boundary and develop a solution using the method of matched asymptotic expansions (e.g., van Dyke 1964) in the limit of small $\eta$. We emphasize that our analysis is inapplicable to an ideal plasma-because standing wave eigenfunctions can develop only for nonzero $\eta$ - but covers the physically relevant case of finite, but arbitrarily small, resistivity. We remark that, although eigensolutions can be obtained from hypergeometric series solutions to equation (3.3), we find it more instructive physically (see Hassam 1991) to develop the boundary layer approach.

We first introduce the parameter

$$
\mu^{2}=\lambda^{2}+m^{2}=\alpha^{2}-k^{2}-2 i \alpha \omega .
$$

Since both the radial wavenumber $k$ and the decay rate $\alpha$ become small in the limit of small $\eta, \mu$ is a small quantity. Therefore it is convenient to rewrite equation (3.3) as

$$
r\left(r f^{\prime}\right)^{\prime}=\frac{\mu^{2}+m^{2} \lambda \eta / r^{2}}{1+\lambda \eta / r^{2}} f
$$

and to develop a solution in the limit of small $\mu$.

For the case $m=0$ only one length scale appears in the problem: it follows that the diffusion layer can be magnified via the transformation $z=r /(\lambda \eta)^{1 / 2}$. Then $|z|<1$ defines the diffusion region and a matched asymptotic expansion can be constructed by extrapolating the inner diffusion layer solution into the wave region $|z| \gg 1$. For nonradial modes a second length scale, determined by $r=\left|(m / \mu)(\lambda \eta)^{1 / 2}\right|$, exists in equation (3.5), which turns out to define the limit of the dissipation region in this case; nothing significant happens in the inner region $r<\left|(\lambda \eta)^{1 / 2}\right|$. Therefore for $m \neq 0$ we invoke the transformation $z=r /\left[(m / \mu)(\lambda \eta)^{1 / 2}\right]$ so that $|z|=1$ again defines the extent of the diffusion region.

To extrapolate the inner solution into the wave domain, we must use an equation valid in both regions. The full equation, approximated only by neglecting $|\lambda \eta| / r^{2} \ll 1$ for $m \neq 0$, is

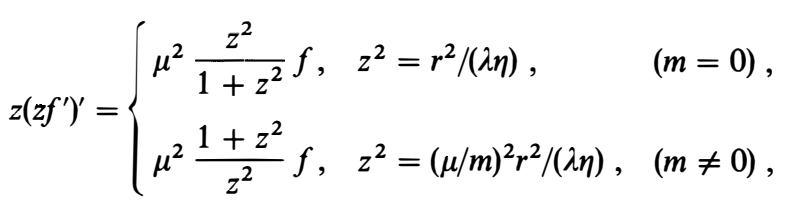

where $f^{\prime}$ now denotes $d f / d z$. The technique of matched asymptotic expansions requires that the inner solution extrapolated into the region $|z|>1$ be matched to the outer wave solution by fixing $|z| \gg 1$ and taking the limit of small $\eta$.

The outer solution is given by

$$
f(z)=\sinh (\mu s), \quad s=-\ln r,
$$

to within an arbitrary normalization. This is just the wave solution, valid for all $m$, obtained by setting $\eta=0$ in the fundamental eigenequation (3.3). It is interesting that the radial wavenumber $k$ is immediately determined by matching this expression to the zeroth-order solution in the inner region, $f=\delta_{m 0}$. However, more structure must be retained in the inner solution to determine the damping rate $\alpha$. Our strategy here is to adopt the simplest analysis adequate to treat the problem. For the purpose of extrapolation of the inner solution into the outer region, it suffices to substitute the crudest approximation to the inner solution, $f=\delta_{m 0}$, into the right-hand side of equation (3.6) and integrate outward from $|z|=1$. To sufficient accuracy (again adopting a minimalist policy), the result is

$$
\begin{aligned}
& f(z)=1, \quad f^{\prime}(z)=\frac{1}{2} \mu^{2} \ln \left(1+z^{2}\right) / z, \quad(m=0), \\
& f(z)=\ln (z), \quad f^{\prime}(z)=1 / z, \quad(m \neq 0) .
\end{aligned}
$$

This extrapolation, which is to be applied in the region $|z|>1$, 
reduces to the boundary value $f=\delta_{m 0}$ (the zeroth order inner solution) on its inner edge $|z|=1$.

We now equate the gradients $d \ln f / d r$ of the inner and outer solutions at some $|z| \gg 1$ to obtain the matching condition

$$
-\mu \ln z= \begin{cases}\operatorname{coth}(\mu s), & (m=0), \\ \tanh (\mu s), & (m \neq 0) .\end{cases}
$$

For small $\mu$ the left-hand side is small, so we invert the hyperbolic functions using $\tanh ^{-1}(x) \approx x+i n \pi$ for small $x$, and, for $m=0$, exploit the identity $\operatorname{coth}^{-1}(x)=\tanh ^{-1}(x) \pm i \pi / 2$. Since, to first order, $\mu=i k-\alpha \omega / k$, the imaginary part of the matching condition gives

$$
k s_{c}=n^{*} \pi, \quad \text { where } n^{*}=n+1-\frac{1}{2} \delta_{m 0}, \quad n=0,1,2, \ldots,
$$

while the real part yields

$$
\alpha=\frac{k^{3}-}{4 n^{*} \omega}, \quad \text { where } \omega^{2}=k^{2}+m^{2}
$$

Here $s_{c} \equiv-\ln r_{c}$ is only strictly defined by the matching condition in the asymptotic limit of small $\eta, s_{c} \rightarrow \frac{1}{2}|\ln \eta|$. Greater accuracy can be obtained, however, by retaining the full scaling which arises naturally from the boundary layer analysis, viz.,

$$
s_{c} \approx \begin{cases}\frac{1}{2}|\ln \omega \eta| & (m=0) \\ \frac{1}{2}\left|\ln (m / k)^{2} \omega \eta\right| & (m \neq 0)\end{cases}
$$

which will be discussed further in $\S 3.4$.

The amazingly simple formula (3.11) for the damping rate $\alpha$ expresses our central result: the rate is quadratic in $|\ln \eta|$ for $m=0$ and cubic for the nonradial modes. Hence, for any fixed $m$, the decay rate is always fast!

\subsection{Physical Interpretation of the Eigensolution}

It is a remarkable fact that much of the essence of the magnetic diffusion problem is contained in the advection region solution (3.7). For small enough damping, we can identify the real and imaginary parts,

$$
f_{\mathbf{R}}=\sin k s, f_{\mathrm{I}}=\frac{\alpha \omega}{k^{2}} k s \cos k s
$$

as the dominant components of the outer eigenfunction. The imaginary component, together with a portion of the real component of similar amplitude, constitutes a traveling wave that bleeds energy from the standing wave into the dissipation region. The amplitude of the imaginary component, $\alpha \omega / k^{2} \propto k$ [eq. (3.11)], becomes vanishingly small as the radial wavenumber is reduced. It follows that the presence of the imaginary component can be taken as a signature for resistive dissipation. The standing wave amplitude vanishes (in the case of nonradial modes) at the boundary of the diffusion region $s=s_{c}$, whereas the imaginary part of the eigenfunction peaks here.

Why is the higher $m$ decay rate given by equation (3.11) slower than the rate for $m=0$ by a factor of $k / m$ ? The wave packet analysis of $\S 3.2$ shows that at moderate $m$ the wave spirals in to the origin taking a time of order $\mathrm{m} / \mathrm{k}$ longer to reach the dissipation region than the purely radial mode. Thus the lengthening of the transit time accounts for the slower decay.

The wave packet description also allows a more accurate determination of the radial wavenumber in the case of large $m$ but fixed $\eta$. This case is not accurately described by the boundary layer analysis except in the limit $\eta \rightarrow 0$. Clearly we would like to determine the $m$-dependence of $s_{c}$ for all $m$ up to the static diffusion limit.

We consider solution (2.12) in the limit $m \gg k$ so that the motion of the wave packet is almost circular. Multiplying solution (2.12) by the damping factor $e^{-\alpha t}$, we determine $\alpha$ by substituting the resulting expression into the exact equation (2.7), regarding $\eta / r^{2}$ as fixed. For small damping rates we find that $\alpha=\frac{1}{2} m^{2} \eta / r^{2}$, and so

$$
\langle\psi\rangle=-\frac{1}{2} m^{2} \eta \dot{r}^{2}\langle\psi\rangle
$$

describes the resistive attenuation of a wave packet in a nearcircular orbit. The inward motion of a wave originating in the outer field comprises a multiplicity of such orbits. Since $\dot{r} / r=$ $k / \omega$ and $\phi=m / \omega$ we can write $\psi=(d \psi / d r) \dot{r}$ and integrate over $r$ to obtain

$$
\ln \langle\psi\rangle=\frac{m^{2} \omega \eta}{4 k r^{2}} \text { for } r \ll 1,
$$

where we have taken $\langle\psi\rangle=1$ at $r=1$. Defining the onset of the diffusion region to be the radius at which the amplitude of the wave packet has decreased by a factor of $e$, we find $r_{c} \approx$ $\left(m^{2} \omega \eta / 4 k\right)^{1 / 2}$, or

$$
s_{c}=\frac{1}{2}\left|\ln \left(m^{2} \omega \eta / 4 k\right)\right|,
$$

a result valid for all physically relevant $\eta$. This value of $s_{c}$ can be used iteratively in the asymptotic formula (3.10) to obtain an improved approximation to $k$ for $m \neq 0$.

We conclude that the distance from the neutral point at which dissipation occurs increases by a factor of approximately $m / k^{1 / 2}$ for the higher $m$ modes. This is due to the grazing angle at which wave packets circulating around the neutral point encounter the diffusion region. Dissipation occurs in an annulus surrounding the neutral point: nonradial perturbations never reach the innermost region where purely radial modes are dissipated.

\subsection{Numerical Results}

We now have a complete analytic description of the dissipation of both radial and nonradial modes. The radial wavenumber $k$ and the decay rate $\alpha$ are computed from (3.10) and (3.11), where the boundary of the diffusion region, $s_{c}$, in given by (3.12) or (3.16), or more simply by $s_{c} \approx \frac{1}{2}|\ln \eta|$, which holds in the asymptotic limit $\eta \rightarrow 0$. Now we will see how well these asymptotic results describe computational solutions at moderate plasma resistivities (typically in the range $10^{-24}<\eta<$ $10^{-3}$ ). We use the more accurate expression (3.16) for $s_{c}$ here, adopting an iterative procedure to determine selfconsistent values of $k, \omega$, and $s_{c}$. For the radial mode we take $s_{c}=$ $\frac{1}{2}|\ln \omega \eta|$.

The computational results presented in Figure 1 confirm that the analytic description is remarkably accurate for all $m, n$, and $\eta$ for which the assumption $|\mu| \ll 1$ holds. Figure $1 a$ compares the analytically and numerically derived values of the radial wavenumber $k$, and Figure $1 b$ does the same for the damping rate $\alpha$. Deviations are apparent only for large $k$ or $\alpha$ which clearly violate the asymptotic nature of the analysis. (In fact, the condition $|\mu| \lesssim 1$ appears to be sufficient to guarantee accuracy.) Plasmas of astrophysical interest ( $\eta \lesssim 10^{-8}$, say) are very accurately described.

It is instructive to compare the decay rates of the radial $(m=0)$ and first nonradial $(m=1)$ modes at modest $\eta$. Dis- 

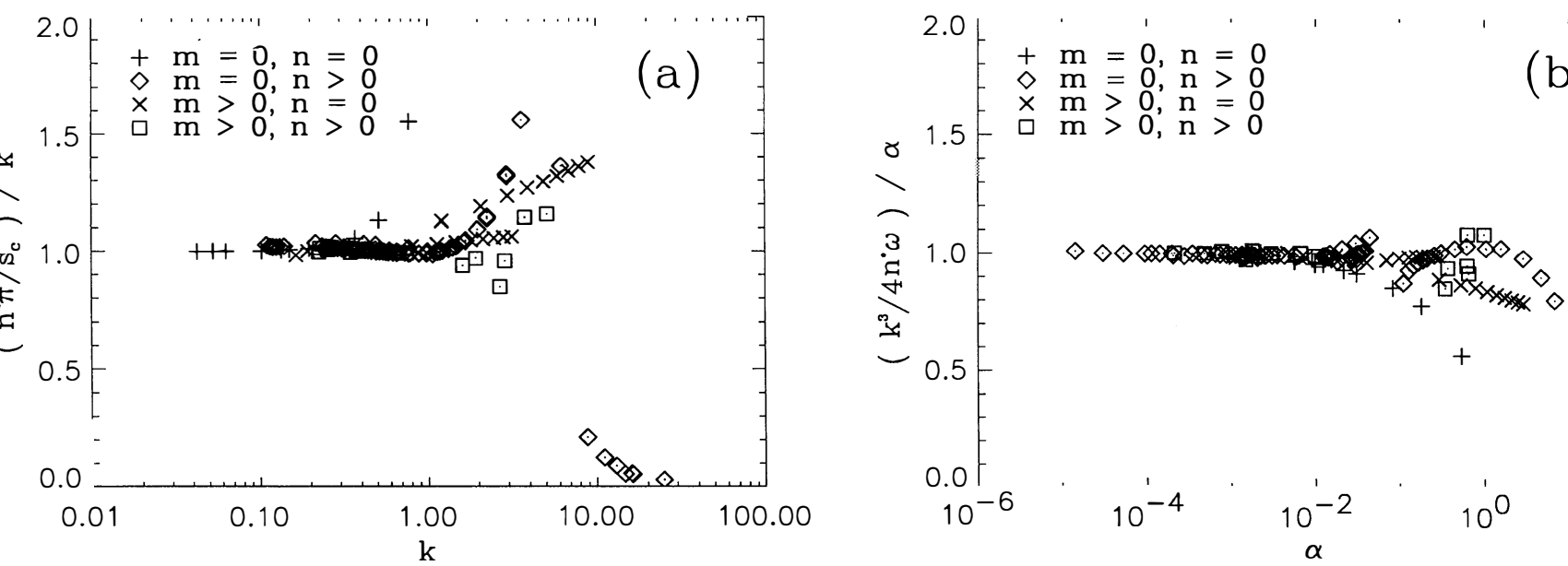

(b)

FIG. 1. Comparison of the asymptotic $(\eta \rightarrow 0)$ scalings obtained analytically in $\S 3.3$, with numerical results for moderate $\eta\left(10^{-24}<\eta<10^{-3}\right)$. In panel $(a)$ the analytic result (3.10), $k=n^{*} \pi / s_{c}$, with $s_{c}$ determined by eq. (3.16) [or (3.12) for $m=0$ ], is compared with numerical solutions of the eigenmode equation (3.3), for a variety of values of $\eta, m$, and $n$. In panel $(b)$, the analytic result (3.11), $\alpha=k^{3} / 4 n^{*} \omega$, is similarly compared. The analytic results are remarkably accurate except in cases of heavy damping, $\eta \gtrsim 10^{-6}$, which clearly violate the assumptions of the analysis.

tinguishing the two cases by a subscript $m$ on $\alpha$ and $k$, we have that for the fundamental radial oscillation $(n=0) k_{1} \approx 2 k_{0}$ from equation (3.10) (neglecting the small diffierence in $s_{c}$ ). Then since $\alpha_{0} \approx k_{0}^{2} / 2$, while $\alpha_{1} \approx k_{1}^{3} / 4$, we find that $\alpha_{1}>\alpha_{0}$ provided $k_{0}>\frac{1}{4}$. It is interesting that for sufficiently large $\eta$ $\left(\eta=e^{-\pi / k_{0}} \gtrsim 3 \times 10^{-6}\right)$ the decay rate of the $m=1$ mode (and the other moderate- $m$ modes) exceeds the decay rate of the fundamental reconnective mode!

This behavior is clearly confirmed in Figure 2, which illustrates the decay rate of the $n=0$ mode as a function of azimuthal node number for diffierent resistivities. A slow decline in the efficiency of dissipation with increasing $m$ is seen, consistent with the $m^{-1}$ dependence in equation (3.11) $(\omega \approx m$ for $m^{2} \gg k^{2}$ ). For modes which are highly oscillatory in the azimuthal direction, the decay rate climbs again toward the static diffusion limit, $\alpha \approx m^{2} \eta$ (eq. [2.11]). The minimum decay rate can be estimated by finding the crossover point between equa-

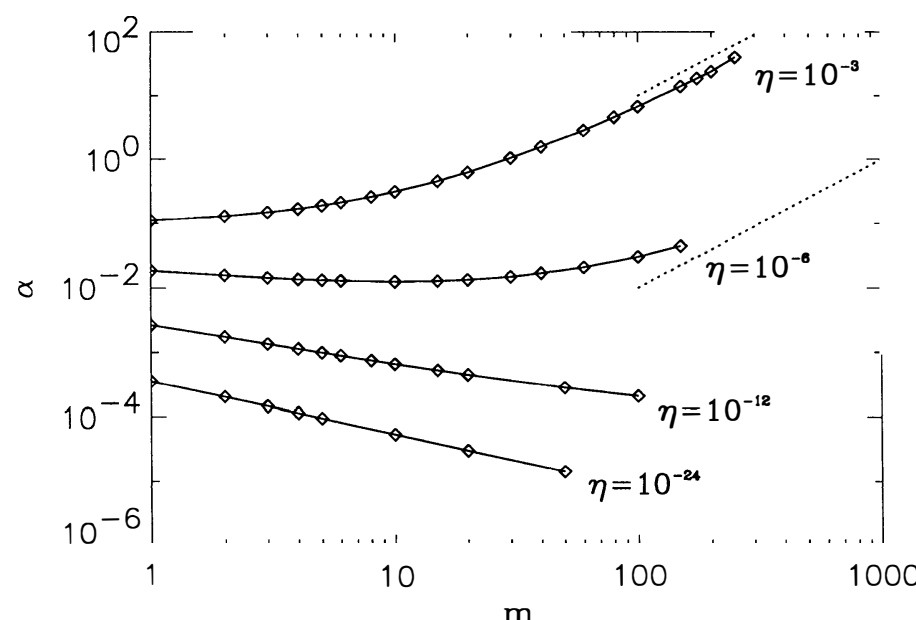

FIG. 2.-The decay rate of $\alpha$ of $n=0$ nonradial modes as a function of the azimuthal node number, $m$, from numerical solutions of the eigenmode eq. (3.3). As shown analytically in $\S 3.6$, the decay rate increases monotonically with $m$ for $\eta \gtrsim 3 \times 10^{-6}$. For $\eta \lesssim 10^{-6}$, the minimum decay rate occurs at $m=O\left(\eta^{-1 / 3}\right)$. For large $m$ the static diffusion limit (2.11) (dotted line) is approached. tions (2.11) and (3.11), yielding

$$
\alpha_{\min } \approx k^{2} \eta^{1 / 3} \text { at } m \approx k / \eta^{1 / 3} .
$$

This decay rate appears "slow" only because here we are fixing $\eta$ and allowing $m$ to vary. The correct scaling requires that $m$ be fixed as $\eta \rightarrow 0$.

\section{LIMITS OF VALIDITY OF THE EIGENMODE SOLUTIONS}

\subsection{Introduction}

The analytic treatment of fast dissipation presented here is made possible only by our assumptions that gas pressure and any longitudinal magnetic field can be neglected, and that the amplitude of magnetic disturbances is small. Here we examine briefly these restrictions. A fuller account of these effiects in the linear and nonlinear regimes will be presented by McClymont \& Craig (1993).

\subsection{Gas Pressure}

The most obvious way in which gas pressure could affiect the progress of reconnection is that, in our closed system, the accumulation of plasma in the outflow lobes may exert a backpressure blocking the entry of fresh magnetic flux to the diffusion region. In fact, the pressure gradient which blocks reconnection forms on the size scale of the diffusion region, underscoring the dynamic nature of the reconnection process. To estimate the magnitude of the gas pressure force, we insert the velocity field resulting from the magnetic force $\boldsymbol{F}_{\boldsymbol{M}}=\boldsymbol{J} \times \boldsymbol{B}$ into the continuity equation $\dot{\rho}=-\nabla \cdot v$, obtaining $\ddot{\rho}=-\nabla \cdot$ $\boldsymbol{F}_{M}$, so to first order the gas pressure force, $\boldsymbol{F}_{G}=-\beta \nabla \rho$ (where $\beta$ is $\gamma / 2$ times the usual plasma $\beta$ ), is related to the magnetic force by

$$
\ddot{\boldsymbol{F}}_{\boldsymbol{G}}=-\beta \boldsymbol{\nabla}\left(\boldsymbol{\nabla} \cdot \boldsymbol{F}_{\boldsymbol{M}}\right) .
$$

To establish the point in space at which these forces are most important, we note that the current density in an inwardmoving disturbance builds up as $r^{-2}$ until the diffusion region is encountered (see, e.g., $\S 2.5$ ), while the background magnetic field varies as $r$, so the magnetic force $\boldsymbol{J} \times \boldsymbol{B}$ peaks near the boundary of the diffusion region. Equation (4.1) suggests that 
the gas pressure force then builds up faster than the magnetic force, allowing the possibility of reconnection being quenched.

Taking $r_{c} \approx(\omega \eta)^{1 / 2}$ as the scale length in equation (4.1), and on the left-hand side $d / d t \approx \omega$, we obtain

$$
F_{G} \approx \frac{\beta}{\omega^{3} \eta} F_{M}
$$

The dominant scaling suggests that the backpressure becomes significant when $\beta \approx \eta$, a result confirmed by numerical simulation (McClymont \& Craig 1993; see also Craig \& Watson 1992).

\subsection{Longitudinal Magnetic Field}

A similar analysis can be performed in the case of a $B_{z}$ component of the magnetic field. Then

$$
\dot{v}=J \times B-\nabla P_{M}, \quad \text { where } \dot{P}_{M}=-\frac{1}{2} B_{z}^{2} \nabla \cdot v+B_{z} B_{E} \cdot v_{z},
$$

and $v$ and $\boldsymbol{B}_{E}$ are the components of velocity and equilibrium magnetic field in the $x-y$ plane. The first term contributing to the build-up of magnetic pressure $P_{M}$ clearly has the same form as that of the gas pressure, while the second term represents shearing of the $x-y$ magnetic field by a flow in the $z$-direction. Since we expect to have $\left|v_{z}\right| /|\boldsymbol{v}| \approx\left|B_{z}\right| /|B|$, the second term should be of comparable magnitude to the first. Therefore we must expect fast reconnection to be inhibited by the presence of a longitudinal magnetic field of magnitude $\frac{1}{2} B_{z}^{2} \gtrsim \eta$. Detailed simulations confirm that the reconnection stalls for finite $B_{z}$ (McClymont \& Craig 1993).

\section{COMPARISON WITH PREVIOUS STUDIES}

\subsection{Introduction}

Here we briefly comment on the relationship of the eigenmode analysis to the initial value problem as discussed by Craig \& Watson (1992), the slowly decaying similarity solution found by Hassam (1991), and the nonlinear theory developed by Petschek (1964). First, we show that the eigenmode analysis is consistent with the spectacular energy release rate found by Craig \& Watson (1992) for pulse-like perturbations in the outer field; then we demonstrate that the evolution of such a pulse may be convincingly reproduced by superposition of eigenmodes.

\subsection{Rapid Dissipation of High-n Radial Modes}

Craig \& Watson (1992) show that a pulse, initially localized in the outer field, undergoes a transient implosion phase which is capable of resistively dissipating the bulk of the free energy of the wave on its first passage through the neutral point. This suggests a much faster time scale than the asymptotic rate found for the fundamental eigenmode. We show that in fact only rather low-order radial harmonics need to be invoked to reproduce this spectacular result.

We first note that the energy decay time $\tau_{\epsilon}$ is half the eigenfunction decay time $1 / \alpha$. So, in relation to the period $\tau_{0}=$ $2|\ln \eta|$ of the fundamental radial eigenmode $(n=0, m=0)$, equation (3.11) implies that the energy decay time of a higher $n$, $m=0$ mode is given by

$$
\frac{\tau_{\epsilon}}{\tau_{0}}=\frac{\tau_{0}}{(2 \pi)^{2}}(1+2 n) .
$$

For a substantial fraction of the energy to be dissipated in the first impact on the neutral point, we require $\tau_{\epsilon} \lesssim \tau_{0} / 4$, which for coronal resistivities of $10^{-12} \lesssim \eta \lesssim 10^{-15}$ is attained for $2 \lesssim n \lesssim 3$. A similar analysis of the nonradial modes yields the condition $n \gtrsim 4(m)^{1 / 2}$ for dissipation in a time $\tau_{0} / 4$ (assuming coronal resistivities and $m \gg k$ ).

If disturbances are generated on the Alfvén time scale at the outer boundary, energy will indeed be concentrated in the higher harmonics which are easily dissipated. Such disturbances have wavelengths of order unity in $s$-space, which from equation (3.10) matches a harmonic $n \approx 2 s_{c} \approx|\ln \eta| \approx 30$ for typical coronal resistivities, far above the threshold for dissipation in one Alfvén transit time, $\tau_{0} / 4$. Nonradial disturbances with the same radial wavenumber and $m \lesssim 50$ will also be dissipated in the first transit of the pulse.

\section{3. "Completeness" of the Eigenmodes}

Since the eigenmode equation (3.3) is not Sturm-Liouville, completeness of the eigenfunctions cannot be guaranteed. In fact, the approximate "similarity" solution (Hassam 1991), in which the current density evolves according to equation (2.13), decaying arbitrarily slowly for small $r$, cannot be described by the eigenmodes, for which the slowest decay is that of the fundamental radial mode. The similarity mode, however, is confined to the diffusion region, so it is both energetically and physically unimportant (as implied by Hassam 1991, and discussed in $\S 2.5$ ). This suggests that the eigenmodes may be able to reproduce the behavior of all physically significant initial conditions, a hypothesis which we test empirically below.

Our aim is to construct an eigenmode representation of the evolution of an azimuthally symmetric $(m=0)$ pulse initially localized in the outer field, and compare it with the evolution determined by integrating the time-dependent equation (2.7) (Craig \& Watson 1992). For each radial node number, $n$, there are two independent eigenmodes, $\psi_{n}(r, t)=\exp \left[\left(i \omega_{n}-\alpha_{n}\right) t\right] f_{n}(r)$, and its complex conjugate, $\psi_{n}^{*}(r, t)=\exp \left[\left(-i \omega_{n}-\alpha_{n}\right) t\right] f_{n}^{*}(r)$. Only the real part of the solution is of physical interest; it turns out that this can be described by a series involving $\psi_{n}(r, t)$ only:

$$
\operatorname{Re}[\psi(r, t)]=\operatorname{Re}\left[\sum_{n=0}^{\infty} c_{n} f_{n}(r) e^{\left(i \omega_{n}-\alpha_{n}\right) t}\right]
$$

The (complex) coefficients $c_{n}$ are obtained by employing GramSchmidt orthogonalization of the two sets of functions $f_{\mathbf{R n}_{n}}(r)$ and $f_{\mathrm{In}}(r)$ to fit specified initial conditions,

$$
\psi(r, 0)=\operatorname{Re}\left[\sum_{n=0}^{\infty} c_{n} f_{n}(r)\right],
$$

and

$$
\frac{\partial \psi(r, 0)}{\partial t}=\operatorname{Re}\left[\sum_{n=0}^{\infty}\left(i \omega_{n}-\alpha_{n}\right) c_{n} f_{n}(r)\right] .
$$

Figure 3 shows an example of the evolution of a perturbation in $\psi$ localized in the outer field, and of zero initial velocity (so $\psi=\eta \nabla^{2} \psi$ at $t=0$ ), with $\eta=10^{-3}$. In the upper panel the evolution was computed by integrating the partial diffierential equation (2.7) in the manner of Craig \& Watson (1992). The rearmost curve shows the initial profile of $\Psi$. In the second curve, the pulse is broadening, and in the third curve it has no split into inward- and outward-propagating parts. By the fifth curve, the outward-moving pulse has reflected off the outer wall (with reversed sign), and it subsequently follows the intially inward-moving pulse toward the diffusion region sur- 


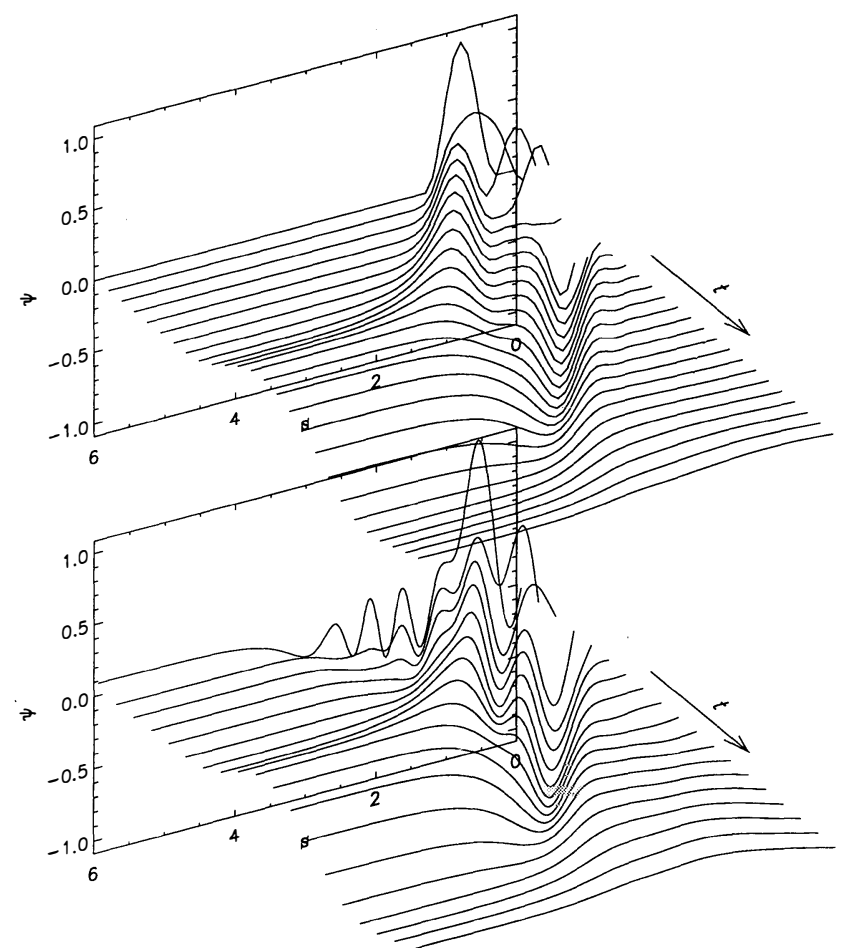

Fig. 3.-Comparison of the evolution of an azimuthally symmetric pulse, initially localized in the outer field, for $\eta=10^{-3}$, as computed by integrating the time-dependent eq. (2.7) (top) and by superposing eigenmodes [eq. (5.2)] (bottom). The outer boundary, $s=0$, is on the right, and the central diffusion region on the left extends to $s \approx 3$. Although the limited number of eigenmodes included $(n=0,1, \ldots, 9)$ cannot reproduce the time-dependent solution exactly, the main features of the evolution are reproduced satisfactorily, including splitting of the initial stationary pulse into two, reflection of the outwardmoving component off the boundary, and dissipation of the inward-moving pulses at the onset of the diffusion region.

rounding the neutral point. A qualitatively similar evolution is seen in the lower panel, which shows a superposition of the first 10 radial eigenmodes, $n=0,1, \ldots, 9$. Despite the obvious presence of Gibbs overshoot, the general features of the evolution seem satisfactorily accounted for, including the splitting of the initial pulse into inward- and outward-propagating waves, reflection of the outward-moving pulse from the boundary, and damping of the pulses when they reach the diffusion region, $s \approx 3$.

\subsection{Comparison with the Petschek Model}

It is instructive to compare the linear theory presented herewith the steady state Petschek reconnective model, which also exhibits fast dissipation. There are many similarities, but significant differences emerge when we consider the extrapolation of the linear analysis into the nonlinear regime.

Petschek (1964) demonstrates that a steady state wave solution can be constructed in which the length and thickness of the current sheet scale as $\eta$. Therefore the aspect ratio of the sheet is independent of $\eta$ while the area of the sheet, $A_{s}$, scales as $\eta^{2}$. The current density in the sheet scales as $\eta^{-1}$, so the flux transfer rate at the neutral point, $\psi=\eta J$, is invariant, but the ohmic dissipation rate is slow since $\eta J^{2} A_{s}$ scales as $\eta$. Thus in Petschek's mechanism the excess magnetic energy is converted not into ohmic heating but into kinetic energy of mass motion, with the outflowing material on the reconnected field lines expelled from the system as a magnetic sling shot.
The linear model is also most effiective as a method for flux annihilation. Here reconnection proceeds via a cylindrical current structure whose area scales linearly with $\eta$ rather than quadratically as in the Petschek model. Free magnetic energy is converted to kinetic energy in one quarter cycle of the oscillation (with $m=0, n=0$ ), on a $|\ln \eta|$ time scale, as in Petschek's model. The time scale for ohmic damping, however, is fast also, scaling as $|\ln \eta|^{2}$, unlike the Petschek mechanism.

Another significant difference is found in the flow pattern around the neutral point. As already mentioned, Petschek postulates that fast nonlinear reconnection is achieved via a current sheet of fixed aspect ratio: an inflow converging on the neutral point is then necessary. In contrast, our linear model exhibits a stagnation point flow, with divergent inflow (see also Parker 1973). The current profile is of fixed aspect ratio (actually circular) only because the perturbed field remains small compared to the background field outside the diffusion region. This places a restriction on the perturbation amplitude: it cannot exceed $O(\eta)$ (see Craig \& Watson 1992 and Hassam 1991).

What happens when the perturbation amplitude is too great to maintain cylindrical symmetry? The imploding wave remains well described by the linear analysis (Craig \& Watson 1992 ) in the outer field, but cylindrical symmetry is eventually lost before the diffusion region is reached. The wave becomes nonlinear as it stalls or accelerates in adjacent lobes of the $\mathrm{X}$, as a result of cancelling or reinforcing of the background field by the perturbation. The end result is the dynamic emergence of a current sheet whose length is determined by the amplitude of the perturbation (to within the restrictions imposed by the geometry) but whose thickness depends critically on the plasma resistivity. As DeLuca \& Craig (1992) and Craig \& Watson (1992) point out, this picture is consistent with the emergence of a quasi-one-dimensional current sheet in which flux is "piled up" at the boundary of the diffusion layer (see Forbes \& Priest 1987), and which releases energy by ohmic dissipation rather than as kinetic energy. The resultant current sheet morphology, flow patterns, and mode of energy release are all strongly at variance with Petschek model.

\section{CONCLUSIONS}

We have developed a complete analytic theory of dynamic linear reconnection at an X-type neutral point. Our analysis extends the eigenfunction approach of Craig \& McClymont (1991) and Hassam (1991) to nonreconnective perturbations; it also exploits the "causal" wave packet description used by Craig \& Watson (1992) to explore the evolution of localized initial disturbances. The essential feature common to all these studies is the representation of any disturbance as a superposition of azimuthal modes. Only the $m=0$ component corresponds to topological reconnection, the nonradial modes being associated with wave disturbances in the outer field.

The eigenmode analysis - as opposed to say a direct simulation of the modal equation - is particularly well suited to an analytic description of the problem at sufficiently long times when only the slowly decaying fundamental modes remain. (We exclude the unimportant similarity mode from further consideration.) Either way, we have demonstrated analytically that all modes decay at the "fast" rate

$$
\alpha=\frac{k^{3}}{4 n^{*} \omega} \text { where } \omega^{2}=k^{2}+m^{2} \text { and } n^{*}=n+1-\frac{1}{2} \delta_{0 m} .
$$


Here $k$ is the radial wavenumber which, for sufficiently small plasma resistivities, scales as $1 /|\ln \eta|$. Therefore all modes have a fast $|\ln \eta|$ scaling with the reconnective mode $(\omega \equiv k)$ having I a quadratic rather than cubic dependence. Resistivity is therefore surprisingly efficient, not only at effecting topological change, but also at dissipating the excess magnetic and fluid energy in an arbitrary perturbation. It should also be stressed that the decay rate of the fundamental $n=0$ mode provides only a lower limit to the spectacular energy release that occurs during the first impact of a pulselike disturbance on the neutral point (Craig \& Watson 1992).

The eigenmode analysis suggests that after an initial "shakedown" period a general disturbance develops into a standing wave oscillation. Energy from the standing wave leaks into the dissipation region surrounding the neutral point, carried by a traveling wave of relative amplitude $O(k)$. The longer decay of the nonradial modes is best understood on the wave packet description. The wavespeed of a spiraling wave is the same as that of a radial mode, while its wavevector is directed predominantly in the azimuthal direction, so its transit time to the diffusion region is increased by a factor of $m / k \propto|\ln \eta|$.

A crucial ingredient of the linear theory is the natural collapse to small length scales of global disturbances focused toward the neutral point. This can be expected to occur under very general circumstances-indeed it must occur if fast dynamic reconnection is to be physically viable. Certainly, in the present geometry, the collapse can be arrested and the reconnection stalled by the influence of finite gas pressure and nonplanar field components, but this could be an artifact of the oversimplified flow topology required for an analytic descrip- tion. Independent numerical studies suggest that more general flow topologies may be capable of maintaining fast reconnection even when these effiects are included (Biskamp \& Welter 1980; DeLuca \& Craig 1992).

What then - if anything - can the linear theory tell us about the massive energy release in solar flares? The linear theory suggests that reconnection must be regarded as an intrinsically dynamic process, which has the potential for Alfvénic dissipation rates. Yet we know that for large amplitude disturbances the current structure at the neutral point can no longer remain cylindrical-it must flatten out into a quasi-one dimensional current sheet (Craig \& Watson 1992; DeLuca \& Craig 1992). Fast dissipation then demands a solution in which magnetic flux piles up at the edges of the current layer and energy is released as heat rather than as kinetic energy of mass motion. The morphology of the current sheet and the mode of energy dissipation are quite distinct from the Petschek model of fast reconnection. Although the dynamic linear analysis provides a similar scaling in some respects to the steady state Petschek reconnection model, its extrapolation into the nonlinear regime produces current sheets and flow topologies whose properties are strongly at variance with the Petschek model.

We thank Paul Watson and Adil Hassam for stimulating conversations. This research was supported by NSF grant ATM-9106052 and by NASA grant NAGW-864. A. N. M. also gratefully acknowledges travel assistance from the University of Waikato Research Council which enabled this work to be completed.
Biskamp, D. 1986, Phys. Fluids, 29, 1520

Biskamp, D., \& Welter, H. 1980, Phys. Rev. Lett., 44, 1069

Craig, I. J. D., \& McClymont, A. N. 1991, ApJ, 371, L41

Craig, I. J. D., \& Watson, P. G. 1992, ApJ, 393, 385

DeLuca, E. E., \& Craig, I. J. D. 1992, ApJ, 390, 679

Dungey, J. W. 1953, Phil. Mag., 44, 725

Forbes, T. G., \& Priest, E. R. 1987, Rev. Geophys., 35, 1583

Hassam, A. B. 1991, Plasma Preprint UMLPR 91-046, Laboratory for Plasma

Research, Univ. of Maryland

McClymont, A. N., \& Craig, I. J. D. 1993 in preparation

\section{REFERENCES}

Parker, E. N. 1963, ApJ, 138, 552 1973, J. Plasma Phys., 9, 49

Petschek, H. E. 1964, in AAS-NASA Symp. on the Physics of Solar Flares, ed. W. H. Hess (NASA SP-50), 425

Strauss, H. R. 1990, J. Geophys. Res. 95, 17145

Sweet, P. A. 1958, in IAU Symp. 6, Electrodynamic Phenomena in Cosmical Physics, ed. B. Lehnert (Cambridge: Cambridge Univ. Press), 123

van Dyke, M. 1964, Perturbation Methods in Fluid Mechanics (New York Academic)

Watson, P. G. 1991, M.Sc. thesis, Univ. of Waikato 